\title{
E26 transformation (ETS)-specific related transcription factor-3 (ELF3) orchestrates a positive feedback loop that constitutively activates the MAPK/Erk pathway to drive thyroid cancer
}

\author{
HEMING CHEN $^{1}$, WEIHONG CHEN ${ }^{3}$, XIAOJUN ZHANG ${ }^{1}$, LIN HU $^{1}$, GUOJUN TANG $^{1}$, \\ JUAN KONG $^{4}$ and ZHIWEI WANG ${ }^{2}$
}

Departments of ${ }^{1}$ Endocrinology, ${ }^{2}$ Gland Surgery, and ${ }^{3}$ Nephropathy, Ankang Central Hospital, Ankang, Shaanxi 725000;
${ }^{4}$ Department of General Practice, Zhoukou Central Hospital, Zhoukou, Henan 466000, P.R. China

Received May 30, 2018; Accepted September 27, 2018

DOI: 10.3892/or.2018.6807

\begin{abstract}
Thyroid cancer is the most commonly diagnosed malignancy of the endocrine system, the incidence of which has increased rapidly in the last 30 years. Genetic alterations in pathways, including the mitogen-activated protein kinase (MAPK)/extracellular signal-regulated kinase (Erk) and phosphatidylinositol-3-kinase (PI3K)/protein kinase B (Akt) pathways, are the driving force behind the development of differentiated thyroid cancer cases into aggressive and undifferentiated forms of thyroid cancer. E26 transformation (ETS)-specific related transcription factor-3 (ELF3) belong to the epithelial-specific subfamily of ETS transcription factors and has recently been reported to be involved in various pathophysiological processes. However, the role of ELF3 in thyroid cancer has not yet been investigated. In the present study, data from The Cancer Genome Atlas (TCGA) were analyzed, and it was revealed that ELF3 was overexpressed in patients with papillary thyroid cancer (PTC). Furthermore, the expression of ELF3 was found to be higher in thyroid cancer tissues with a B-Raf proto-oncogene (BRAF) mutation as determined by western blot analysis and IHC staining. Additionally, ELF3 overexpression predicted a poor prognosis in patients with PTC. The MAPK signaling pathway inhibitor PLX4032 was demonstrated to strongly attenuate ELF3 protein levels in BRAF-mutant thyroid cancer cell lines. Knockdown of ELF3 with small interfering RNA (siRNA) inhibited the growth, clone formation, migration and invasion of BRAF mutant thyroid cancer cells. Mechanistically, ELF3 modulated the activity of the MAPK/Erk pathway via transcriptional regulation of the human epidermal growth factor receptor 2 family
\end{abstract}

Correspondence to: Dr Zhiwei Wang, Department of Gland Surgery, Ankang Central Hospital, 85 Jinzhou Road, Hanbin, Ankang, Shaanxi 725000, P.R. China

E-mail: wangzhiwei87865566@126.com

Key words: ELF3, prognosis, BRAF mutation, thyroid cancer, MAPK/Erk pathway, feedback loop of receptors as determined by RT-qPCR. In conclusion, the present study demonstrated ELF3 to be a potential prognostic marker for patients with thyroid cancer. Notably, ELF3 was demonstrated to form a positive feedback loop with MAPK pathways leading to the progression of BRAF-mutant thyroid cancer.

\section{Introduction}

Epithelial follicular cell-derived thyroid cancer is the most commonly diagnosed endocrine malignancy and its incidence has increased 3 -fold over the past 30 years $(1,2)$. Papillary thyroid cancer (PTC) accounts for $>80 \%$ of all cases of thyroid cancer. PTC is usually not aggressive and has a 5-year survival rate of over $95 \%$ (3). However, $10 \%$ of cases develop into more aggressive and undifferentiated forms of thyroid cancer; such cases are characterized by metastasis and resistance to conventional therapy, leading to recurrent disease and mortality (4). Genetic alterations in pathways, including the mitogen-activated protein kinase (MAPK)/extracellular signal-regulated kinase (Erk) and phosphatidylinositol-3-kinase (PI3K)/protein kinase B (Akt) pathways, are the driving force behind thyroid tumorigenesis and progression (5). Previous studies have reported that over $70 \%$ of activating somatic alterations of gene-encoding effectors occur within the MAPK/Erk signaling pathway; mutations in BRAF are the most common cause of aberrant MAPK/Erk signaling (6,7).

In addition to aberrant signaling pathways, other mechanisms such as alterations in E26 transformation (ETS) family members of transcription factors have been implicated in all steps of tumor progression (8). ETS-specific related transcription factor-3 (ELF3) (ELF3, also known as ESE-1) belongs to the epithelial-specific subfamily of ETS transcription factors and has been reported to be involved in various pathophysiological processes, including cancer and inflammatory disorders $(8,9)$. ELF3 was reported to serve an oncogenic role in prostate cancer via constitutive activation of the NF- $\kappa B$ pathway and the formation of a feedback loop with interleukin (IL)-1 $\beta$ (10). However, other studies have reported a contrary role of ELF3; for example, suppression of E2-dependent MCF7 cell proliferation via inhibition of the transcription 
of the estrogen receptor, and increase in the transcriptional activity of tumor growth- and invasion-promoting genes (early growth response protein 1 and TGFBR2) in squamous cancer types by directly binding to the promoter region $(10,11)$. This controversial role of ELF3 suggests that it serves multiple roles in tumorigenesis. Nevertheless, its exact function in thyroid tumorigenesis has not yet been examined.

The present study aimed to examine the exact role of ELF3 in thyroid cancer and the mechanism underlying the ELF3-associated promotion of thyroid cancer progression. Firstly, it was revealed that ELF3 was overexpressed in PTC compared to normal tissue, and an even higher level of ELF3 was demonstrated in BRAF-mutant PTC compared with BRAF wild-type PTC. High levels of ELF3 were found to be associated with a poor prognosis in patients with PTC. ELF3 silencing dramatically inhibited cell growth and invasiveness in BRAF-mutant thyroid cancer cell lines. To the best of our knowledge, the present study was the first to reveal positive feedback loops between ELF3 and the MAPK/Erk signaling pathway, contributing to thyroid tumorigenesis. The results of the present study support the hypothesis that ELF3 functions as an oncogene in thyroid cancer.

\section{Materials and methods}

TCGA data analysis. All the TCGA data were downloaded from Cancer Broswer in UCSC database (https://genome. ucsc.edu/index.html). Normalized mRNA expression (ELF3, EGFR, HER2, HER3 and HER4) of PTC and corresponding clinical data were included in files. Each tumor sample has its own identify number with '01' at the end, while paired normal thyroid sample with ' 11 ' at the end.

Samples. Paraffin-embedded samples [24 papillary thyroid cancer (PTC) specimens and 9 non-neoplastic thyroid specimens] and fresh tissues (17 PTC tissues with paired non-neoplastic thyroid tissues) were obtained by surgery at Ankang Central Hospital and Zhoukou Central Hospital, following institutional review board approval. Written informed consent was obtained from each patient before surgery. All the samples were collected from 30 female and 11 male patients between January 2006 and December 2017. The age of the participants ranging from 30 to 65 years with median age of 53 years. The fresh tissues were cut into cubes and put into sterile freezing tubes. Samples were stored at $-80^{\circ} \mathrm{C}$.

Reagents. The RAF inhibitor PLX4032 was purchased from Selleck Chemicals (Houston, TX, USA) and dissolved in dimethyl sulfoxide (DMSO) for use. BCPAP and 8505C cells were treated with $1 \mu \mathrm{M}$ PLX4032 for $10 \mathrm{~h}$, and a western blot analysis was performed to evaluate the effects of this inhibitor on the MAPK/Erk pathway.

Immunohistochemistry (IHC). Specimens were cut into 5- $\mu \mathrm{m}$ sections. The sections were deparaffinized and rehydrated in a graded series of ethanol, and washed in PBS. The sections were incubated with the anti-ELF3 antibody at a dilution of 1:150 (cat. no. 5715-1; Epitomics-an Abcam Company, Burlingame, CA, USA) at $4{ }^{\circ} \mathrm{C}$ overnight and with a secondary antibody (cat. no. sp9001; Beijing Zhongshan Jinqiao Biotechnology
Co., Ltd., Beijing, China) for 30 min. Diaminobenzidine (DAB) (Beijing Zhongshan Jinqiao Biotechnology Co., Ltd.) was used for visualization. A light microscope (Olympus Corp., Tokyo, Japan) was used to observe and photograph the staining. For expression analysis, the staining was categorized as negative, weak, medium and strong and was confirmed by an in-house pathologist.

Cell culture and siRNA transfection. All human thyroid cancer cell lines (BCPAP, 8505c and TPC-1) and immortalized thyroid epithelial cell line (HTori-3) were obtained from the Shanghai Cell Bank (Type Culture Collection Committee, Chinese Academy of Sciences, Shangai). Cells were cultured in RPMI-1640 medium (cat. no. 1937557; Gibco; Thermo Fisher Scientific, Inc., Waltham, MA, USA) with 10\% FBS (Biological Industries, Shangai, China) and maintained in an incubator with $5 \% \mathrm{CO}_{2}$ at $37^{\circ} \mathrm{C}$. For transient siRNA transfection, cells were seeded into normal growth medium at $30 \%$ confluence in 6-well tissue plates $24 \mathrm{~h}$ prior to transfection with 5 nM siRNA (siELF3-1, 5'-GCUACCAAGUGGAGA AGAATT-3' and siELF3-2, 5'-GCCAUGAGGUACUACUAC ATT-3'; Shanghai GenePharma Co., Ltd., Shanghai, China) using Lipofectamine 2000 reagent (Invitrogen; Thermo Fisher Scientific, Inc.), in accordance with the manufacturer's instructions. Non-specific siRNA (siControl; Shanghai GenePharma Co., Ltd.) was used as a negative control. After $48 \mathrm{~h}$ of transfection, the cells were harvest for subsequent experimentation.

$R N A$ extraction and reverse transcription-quantitative polymerase chain reaction ( $R T-q P C R)$. Total RNA was extracted from fresh samples and cell lines using TRIzol reagent (Takara Biotechnology Co., Ltd., Dalian, China). cDNA was synthesized from $1 \mu \mathrm{g}$ total RNA with a PrimeScript RT reagent kit (Takara). RT-qPCR was performed on a CFX96 Thermal Cycler Dice $^{\mathrm{TM}}$ system (Bio-Rad Laboratories, Inc., Hercules, CA, USA) using SYBR Green (BioTools Pty. Ltd., Queensland, Australia) under the following cycling conditions: $3 \mathrm{~min}$ at $95^{\circ} \mathrm{C}$, followed by 35 cycles of $10 \mathrm{sec}$ at $95^{\circ} \mathrm{C}$ and $45 \mathrm{sec}$ at $58^{\circ} \mathrm{C}$. The mRNA expression of ELF3 was normalized to 18SrRNA. Relative mRNA expression was calculated by using $2^{-\Delta \Delta C q}$ method (12). Each sample was run in triplicate. Primer:18s F: 5'-CGCCGCTAGAGGTGAAATTC-3' R: 5'-CTTTCGCTC TGGTCCGTCTT-3', ELF3 F: 5'-CATGACCTACGAGAA GCTGAGC-3' R: 5'-GACTCTGGAGAACCTCTTCCTC-3', EGFR 5'-AACACCCTGGTCTGGAAGTACG-3' R: 5'-TCG TTGGACAGCCTTCAAGACC-3', HER2 F: 5'-GGAAGT ACACGATGCGGAGACT-3' R: 5'-ACCTTCCTCAGCTCC GTCTCTT-3', HER3 F: 5'-CTATGAGGCGATACTTGGAAC GG-3' R: 5'-GCACAGTTCCAAA GACACCCGA-3'.

Western blot analysis. Cells were lysed in pre-chilled radioimmunoprecipitation assay buffer containing protease inhibitors (Sigma-Aldrich; Merck KGaA, Darmstadt, Germany). Supernatants were collected and loaded onto 10\% SDS-PAGE gels and transferred onto PVDF membranes (Roche). Membranes were blocked with 5\% BSA for $1.5 \mathrm{~h}$ at room temperature. The membranes were then incubated with primary antibodies: Anti-ELF3 (1:750; cat. no. 5715-1; Epitomics), anti-phospho-Akt (dilution 1:1,000; cat. no. BS4007; Bioworld Technology, Inc., St. Louis Park, 
MN, USA), anti-phospho-Erk1/2 (dilution 1:1,000; cat. no. 4370; Cell Signaling Technology, Inc., Danvers, MA, USA), anti-total-Akt (dilution 1:1,000; cat. no. BS1379; Bioworld), anti-total-Erk1/2 (dilution 1:1,000; cat. no. 9102; Cell Signaling Technology) and anti-tubulin (dilution 1:200; cat. no. sc-9104; Santa Cruz Biotechnology, Inc., Dallas, TX, USA). This was followed by incubation with species-specific HRP-conjugated secondary antibodies (cat. nos. 130004 and 130023) from OriGene Technologies, Inc. (Rockville, MD, USA). Samples were visualized using the Western Bright ECL detection system (Millipore Corp., Billerica, MA, USA). The densitometry were analyzed with Tanon Gis 1D 4.2 (Tanon, Shanghai, China).

Cell proliferation assay and colony formation. Cells were seeded at a concentration of 800 cells/well and cultured in 96-well plates for 1, 3, 5 and 7 days following treatment with si-ELF3 or control siRNA for $48 \mathrm{~h}$. At the indicated times, $20 \mu \mathrm{l}$ of $0.5 \mathrm{mg} / \mathrm{ml}$ MTT (Sigma-Aldrich; Merck KGaA) was added into the medium and incubated for $4 \mathrm{~h}$, followed by $150 \mu \mathrm{l}$ DMSO for additional $15 \mathrm{~min}$. A microplate reader (Dynatech Laboratories, El Paso, TX, USA) was used to measure the absorbance using a test wavelength of $570 \mathrm{~nm}$. For clone formation, transfected cells were seeded at a concentration of 1,000 cells/well and cultured in 12-well plates. The medium was refreshed every 3 days. Following 7-10 days of culture, surviving colonies ( $\geq 50$ cells/colony) were fixed with methanol and stained with $0.5 \%$ crystal violet, and the colonies were counted with Image-Pro Plus 6.0 (Media Cybernetics, Rockville, MD, USA). Each experiment was performed in triplicate.

Transwell assays. Cell migration and invasion assays were assessed with Transwell chambers $(8.0-\mu \mathrm{m}$ pore size; Corning Inc., Corning, NY, USA). For the cell invasion assay, Transwell chambers were coated with Matrigel (4X dilution; $15 \mu \mathrm{l} /$ well; BD Biosciences, Franklin Lakes, NJ, USA). Cells were seeded in the upper chamber at a density of $1 \times 10^{4}$ cells $/ \mathrm{ml}$ for the migration assay and $1 \times 10^{4}$ cells/ml for the invasion assay in $200 \mu \mathrm{l}$ medium containing $0.5 \%$ FBS. Medium with $20 \%$ FBS ( $1 \mathrm{ml}$ ) was added to the lower chamber. After a 12 or $24 \mathrm{~h}$ incubation, non-migrating/non-invading cells in the upper chamber were removed with a cotton swab, and migrating/invading cells were fixed in $100 \%$ methanol and stained with crystal violet solution $(0.5 \%$ crystal violet in $2 \%$ ethanol). Images of five fields of view chosen at random were taken for each membrane. The number of migrating/invading cells was expressed as the average number of cells per microscopic field over five fields of view. Images were captured with an inverted Olympus IX71 microscope (Olympus Corp., Tokyo, Japan).

Statistical analysis. The gene expression analysis used data from The Cancer Genome Atlas (TCGA) (URL: https://genome-cancer.soe.ucsc.edu). The median signal intensity was set as the cutoff value of ELF3 overexpression. The linear correlation between the expression of ELF3 and HER family members were calculated with the Pearson's correlation coefficient. Variance analysis was performed to compare the differences between independent groups. $\mathrm{P}<0.05$ was considered to be indicative of a statistically significant result. Results are shown as the mean \pm standard deviation. Kaplan-Meier survival curve analysis was used to assess the survival of PTC patients. Linear regression analysis was used to evaluate the relationship of the ELF3 expression with the expression of HER/ErbB family of receptors. Data analysis was performed with GraphPad Prism (version 5.01; GraphPad Software, Inc., La Jolla, CA, USA).

\section{Results}

Increased expression of ELF3 is a potential prognostic marker for patients with PTC. The expression of ELF3 in normal thyroid and PTC tissues in The Cancer Genome Atlas (TCGA) dataset (13) was analyzed. As presented in Fig. 1A, the mRNA expression of ELF3 was significantly upregulated in primary PTC compared with that noted in the normal thyroid tissue. This was further supported by the TCGA dataset, which revealed that ELF3 expression in PTC was significantly higher compared with that in matched normal thyroid tissues (Fig. 1B). ELF3 expression in 17 PTCs and matched non-cancerous thyroid tissues (control subjects) was analyzed to confirm the results of the database analysis. Consistent with the TCGA dataset, ELF3 expression was also higher compared with that observed in the matched normal thyroid tissues at the mRNA (Fig. 1C) and protein levels (Fig. 1D).

PTC is not usually aggressive and has a 5-year survival of over 95\% (3). The prognostic capacity of the ELF3 expression level for patients with PTC in the TCGA dataset was analyzed using Kaplan-Meier survival curves; median ELF3 expression was set as the cutoff point. The results revealed that the ELF3 expression level was significantly associated with poor survival, and mortality occurred earlier post-diagnosis in the patients with higher ELF3 expression levels (Fig. 1E). Together, these data indicated the oncogenic function and the potential value for prognosis evaluation of ELF3 in thyroid cancer.

BRAF mutation-induced overactivation of the MAPK signaling pathway results in upregulation of ELF3 in thyroid cancer. Mutant BRAF has previously been demonstrated to induce overactivation of the MAPK/Erk pathway in thyroid tumorigenesis (5). Therefore, the association between BRAF mutation and ELF3 expression in the TCGA dataset was investigated. As presented in Fig. 2A, ELF3 expression was significantly higher in BRAF-mutant $\left(\mathrm{BRAF}^{\mathrm{MU}}\right)$ PTC compared with wild-type BRAF $\left(\right.$ BRAF $\left.^{\mathrm{WT}}\right)$ PTC tissues. Further analysis revealed a statistical difference between BRAF ${ }^{\mathrm{WT}} \mathrm{PTC}$ and normal thyroid tissues (Fig. 2B). Western blot analysis was performed to investigate the ELF3 expression pattern in 3 different thyroid cancer cell lines (BCPAP, 8505c and TPC-1) and 1 immortalized thyroid epithelial cell line (HTori-3). The BRAF mutant thyroid cancer cell lines (BCPAP, 8505c) revealed high basal levels of ELF3 compared with the BRAF wild-type thyroid cancer cell line (TPC-1) and non-cancer thyroid epithelial cell line (Fig. 2C). To evaluate the impact of MAPK/Erk signaling on ELF3 expression, two BRAF-mutant cell lines with higher ELF3 mRNA expression were selected and treated with RAF inhibitor PLX4032 at a final concentration of $1 \mu \mathrm{M}$ for $6-8 \mathrm{~h}$. As presented in Fig. 2D, inhibition of MAPK/Erk signaling markedly attenuated ELF3 expression in BRAF-mutant cells. These observations support the hypothesis that ELF3 
A

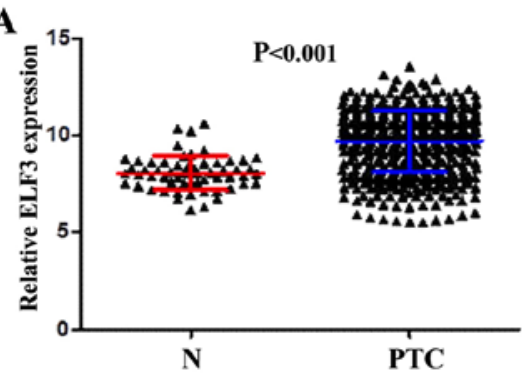

D

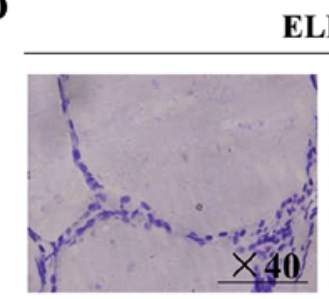

Normal thyroid

ELF3

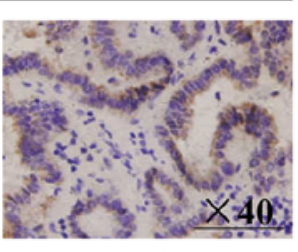

PTC
B

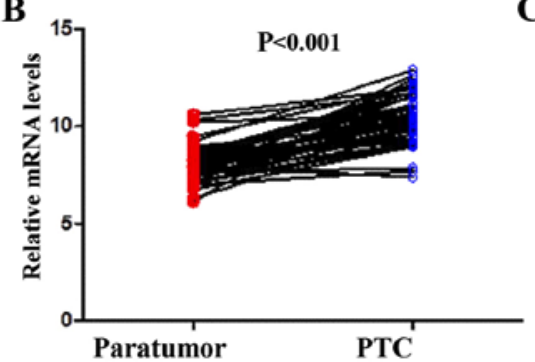

C

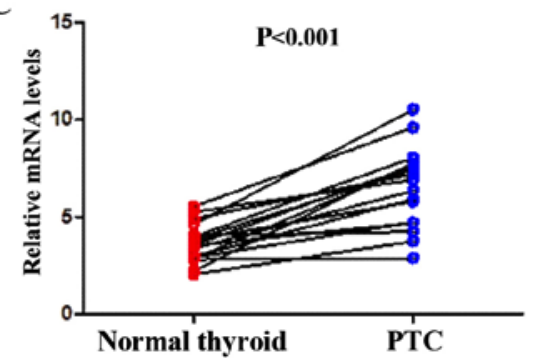

E

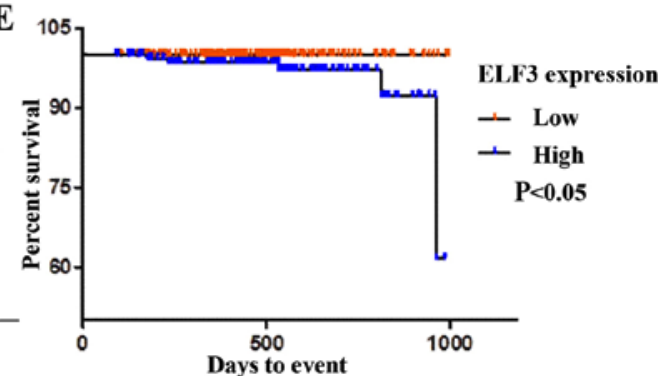

Figure 1. Expression of E26 transformation (ETS)-specific related transcription factor-3 (ELF3) is increased in papillary thyroid carcinoma (PTC) and higher ELF3 expression predicts poor prognosis of patients with PTC. (A) Expression of ELF3 in PTC and normal thyroid tissues (N) in The Cancer Genome Atlas (TCGA) dataset (n=505). (B) Gene expression of ELF3 was significantly higher in PTC compared with matched non-neoplastic thyroid tissues (NT) in the TCGA dataset ( $\mathrm{n}=63)$. (C) Relative ELF3 expression was upregulated in 17 PTC tissues compared with matched normal thyroid tissues as determined by RT-qPCR assay; 18S RNA was used as a normalized control for RT-qPCR assay. (D) Immunohistochemical staining was performed to detect ELF3 expression in 24 PTC and 9 normal thyroid specimens. Representative ELF3 staining and histograms of the percentage of ELF3-positive cells from 5 microscopic fields in each group (right panel) are shown. Statistically significant differences are indicated. (E) Patients with high ELF3 expression exhibited shorter survival times than the patients with low ELF3 expression from The Cancer Genome Atlas (TCGA) dataset ( $\mathrm{n}=334)$. Kaplan-Meier survival curves were used to assess the survival of PTC patients. The median expression level was used as the cutoff point.

A

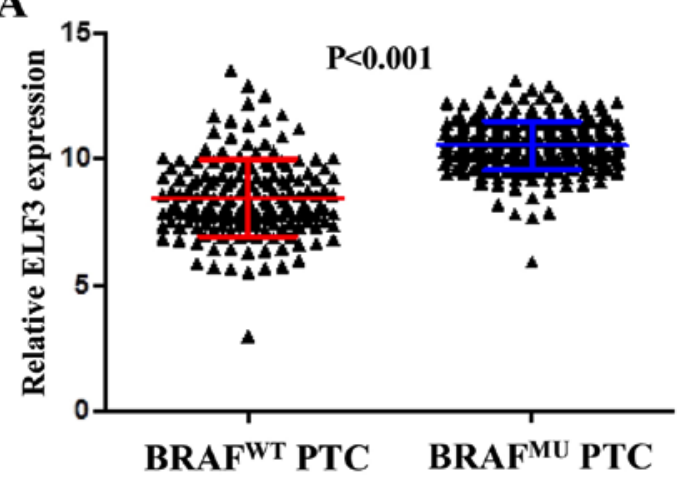

C

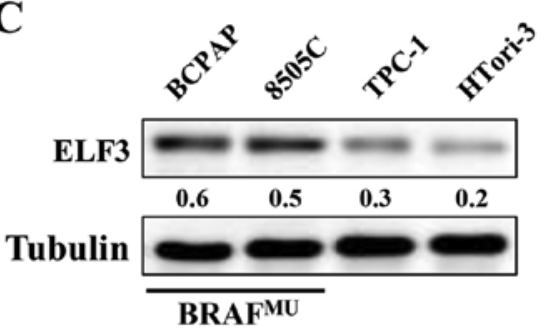

B

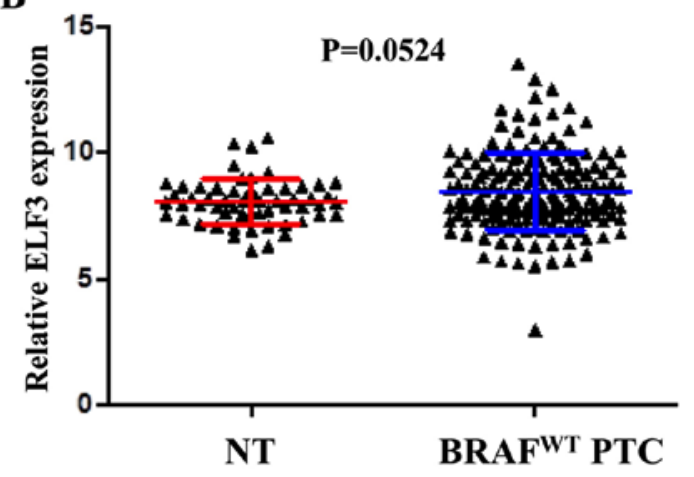

D

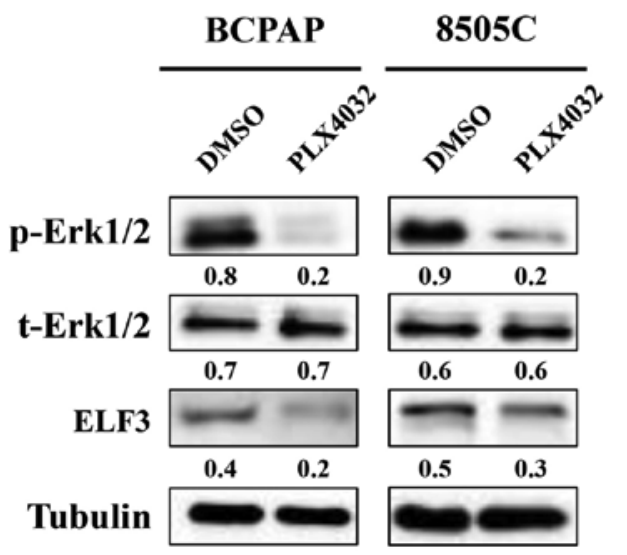

Figure 2. BRAF mutation is associated with overexpression of E26 transformation (ETS)-specific related transcription factor-3 (ELF3) in thyroid cancer. (A) Expression of ELF3 in BRAF wild-type papillary thyroid carcinoma (BRAF ${ }^{\mathrm{WT}} \mathrm{PTC}$ ) and BRAF-mutant PTC (BRAF ${ }^{\mathrm{MU}}$ PTC) in the TCGA dataset. (B) Expression of ELF3 in normal thyroid tissues (NT) and BRAF ${ }^{\mathrm{WT}} \mathrm{PTC}$ in the TCGA dataset $(\mathrm{n}=505)$. (C) Expression of ELF3 in two BRAF-mutant thyroid cancer cell lines (BCPAP, 8505C), one BRAF wild-type thyroid cancer cell (TPC-1) and one immortalized thyroid epithelial cell (HTori-3) was determined by western blot analysis. (D) BCPAP and 8505C cells were treated with $1 \mu \mathrm{M}$ PLX4032 for $10 \mathrm{~h}$. Western blot analysis was performed to evaluate the effects of PLX4032 on the levels of the phosphorylation of Erk and the expression of ELF3. Tublin was used as a loading control. 
A

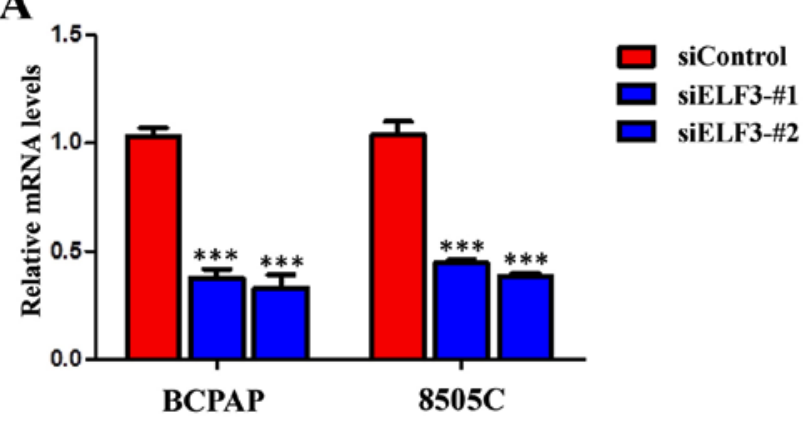

B

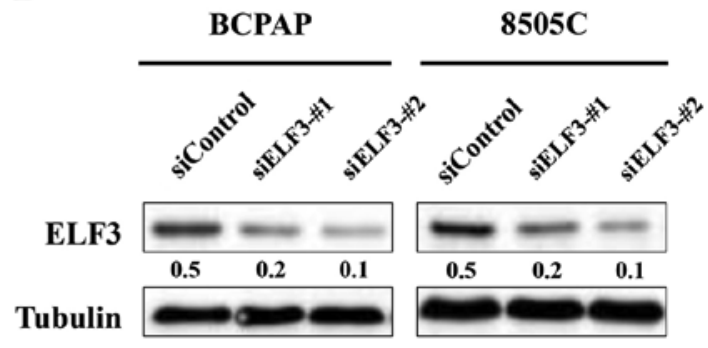

C
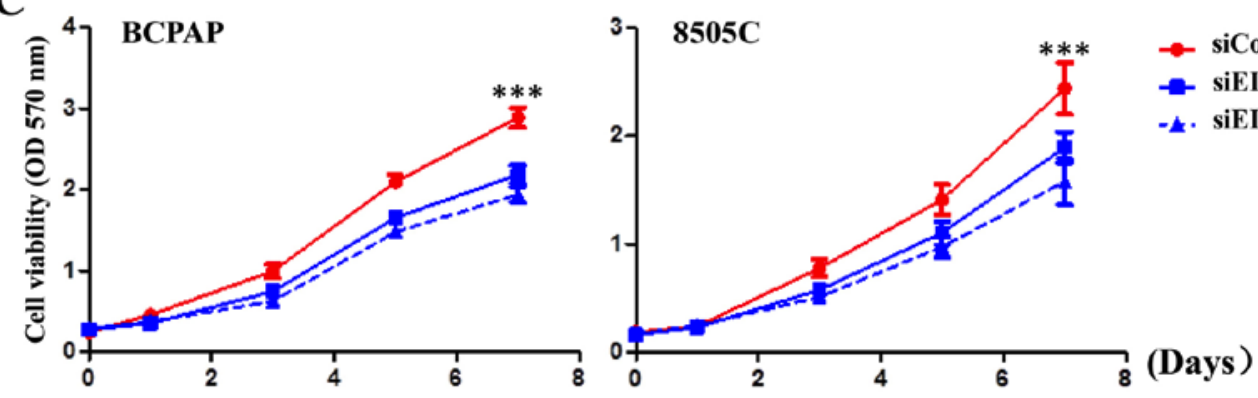

D
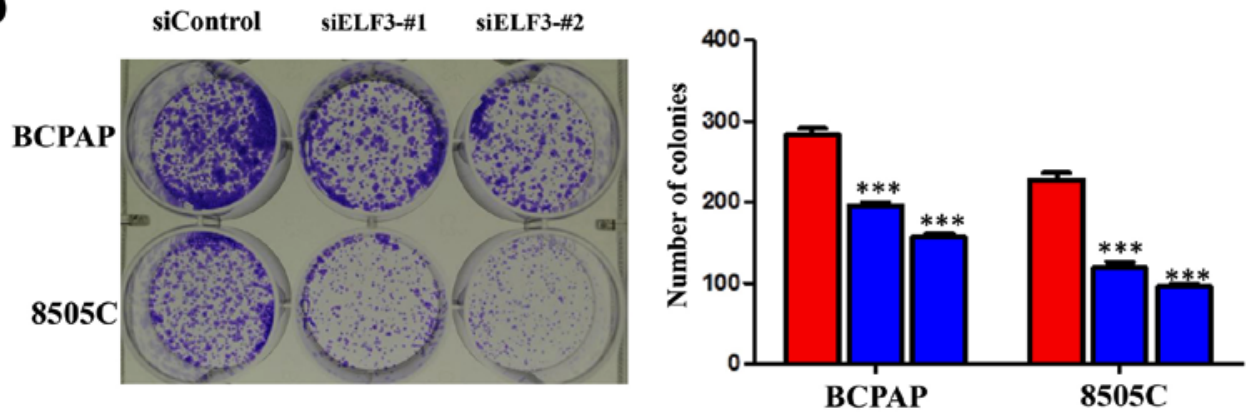

Figure 3. E26 transformation (ETS)-specific related transcription factor-3 (ELF3) promotes thyroid cancer cell growth. Knockdown of (A) ELF3 mRNA and (B) protein with two different siRNAs (siELF3-\#1 and siELF3-\#2) in BCPAP and 8505C cells was evidenced by RT-qPCR and western blot analysis, respectively. 18S RNA was used as a normalized control for RT-qPCR assay, and tubulin was used as a loading control for western blot analysis. (C) ELF3 knockdown significantly inhibited cell viability. (D) ELF3 knockdown significantly inhibited colony formation of thyroid cancer cells. Left panel shows the representative images of colony formation in cells transfected with the indicated siRNAs. Quantitative analysis of colony numbers is shown in the right panel. All data are presented as mean \pm standard deviation. ${ }^{* * *} \mathrm{P}<0.001$.

expression is upregulated by the MAPK/Erk signaling pathway in BRAF-mutant thyroid cancer.

ELF3 knockdown inhibits thyroid cancer cell growth. To illustrate the biological function of ELF3, knockdown experiments were performed in BRAF-mutant thyroid cancer cell lines BCPAP and 8505C. Knockdown of ELF3 by two different siRNA sequences (siELF3-\#1 and \#2) was confirmed at the mRNA level by RT-qPCR (Fig. 3A) and at the protein level by western blot analysis (Fig. 3B). Knockdown of ELF3 significantly inhibited the proliferation and clone formation of BCPAP and 8505C thyroid cancer cell lines compared with the non-sense siRNA control (siControl) (Fig. 3C and D).

ELF3 knockdown inhibits thyroid cancer cell migration and invasion. The majority of cancer-associated mortality is caused by invasion and metastasis (14). Therefore, it was hypothesized that early mortality was correlated with high ELF3 levels in patients with thyroid cancer, resulting in the induction of cancer cell invasion and metastasis. Transwell assays were performed to investigate this hypothesis. As illustrated in Fig. 4A, the number of migrated cells was significantly lower in the siELF3-\#2 transfected cells compared with the siControl transfected cells. Furthermore, the invasion assay demonstrated that ELF3 knockdown significantly decreased the ability of cells to pass through the Matrigel-coated membrane (Fig. 4B). These data suggested a strong link between high expression of ELF3 and metastatic phenotypes in BRAF-mutant thyroid cancer cells.

ELF3 transcriptionally regulates the expression of the $H E R / E r b B$ family of receptors to form a positive feedback loop with MAPK pathways in BRAF-mutant thyroid cancer. It has previously been reported that the HER/ErbB family of receptors, including EGFR, HER2, HER3 and HER4, serves a critical role in the tumorigenesis of thyroid cancer (15-17). Furthermore, certain potential ETS binding sites (EBS, 5'-GGAA/T-3') were revealed in HER/ErbB family promoter regions using MatInspector online software (www.genomatix. de/online_help/help_matinspector/matinspector_help). It 
A
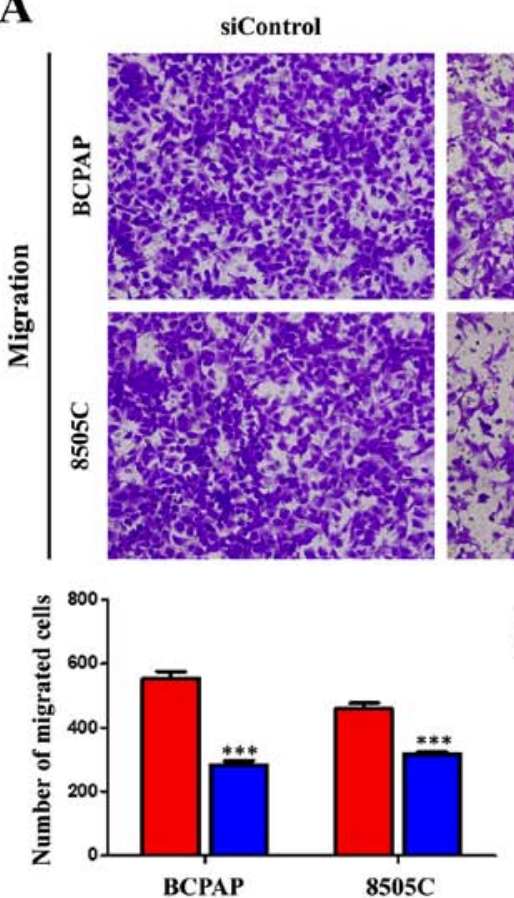

siELF3-\#2

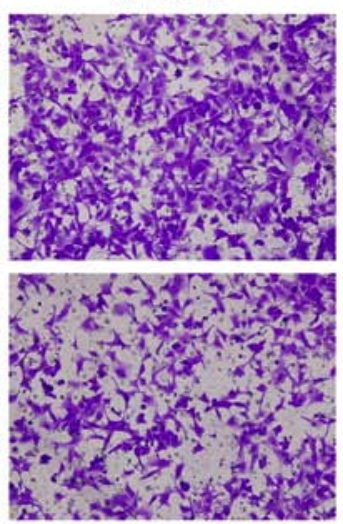

$\square$ siControl
B
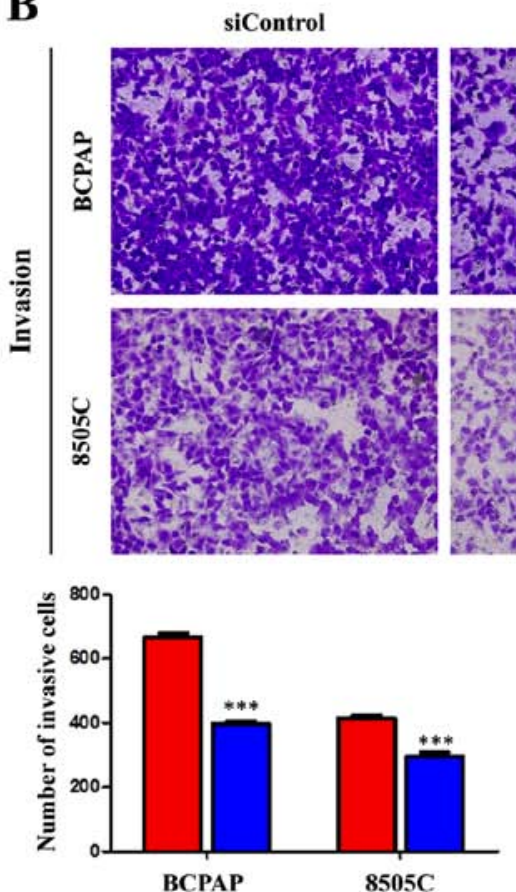

Figure 4. Inhibition of thyroid cancer cell migration and invasion by knockdown of E26 transformation (ETS)-specific related transcription factor-3 (ELF3). Effects of ELF3 knockdown on (A) migration and (B) invasion of BCPAP and 8505C cells was measured by Transwell assays. Results were quantitated by counting migratory and invasive cells in 3 randomly chosen high-power fields for each replicate. Representative fields are shown. All the data are presented as mean \pm standard deviation. ${ }^{* * *} \mathrm{P}<0.001$.

was therefore hypothesized that ELF3, as a member of the ETS transcription factor family, may be associated with the activation of the HER family of receptors. To verify this hypothesis, the association between ELF3 and the HER family in the TCGA dataset was analyzed. The expression of HER family members was observed to have a significant positive association with ELF3 expression, except for HER4 (Fig. 5A). Furthermore, ELF3 knockdown significantly decreased the expression of HER2 and HER3 in BCPAP and 8505C thyroid cancer cell lines (Fig. 5B). A growing body of evidence indicates that overexpression of HER family members leads to the activation of downstream pathways, including MAPK/Erk and PI3K/Akt, which serve a fundamental role in thyroid tumorigenesis and cancer progression $(5,18)$. Therefore, it was hypothesized that high expression of ELF3 induced by over-activation of the MAPK signaling pathway may form a positive feedback loop with the MAPK signaling pathway in BRAF-mutant PTC. As presented in Fig. 5C, ELF3 knockdown attenuated the phosphorylation of Erk (p-Erk) in the BCPAP and $8505 \mathrm{C}$ thyroid cancer cells. However, the phosphorylation of Akt (p-AKT) was not affected. Collectively, these data indicated that overexpression of the oncogene ELF3 is induced by the activated MAPK/Erk pathway, and ELF3 may, in turn, further activate the MAPK/Erk pathway, potentially via transcriptional upregulation of the HER/ErbB family of receptors, in BRAF-mutant PTC.

\section{Discussion}

To the best of our knowledge, the present study is the first to provide evidence to support the oncogenic role of ELF3 in BRAF-mutant thyroid cancer. ELF3 was revealed to be highly expressed in primary PTCs compared with that noted in thematched non-tumor tissues. It was also revealed that increased expression of ELF3 may be used as a potential prognostic marker for PTC patients. Finally, ELF3 was demonstrated to form positive feedback loops with the MAPK/Erk signaling pathway, and therefore contribute to thyroid tumorigenesis.

As a member of the ETS transcription factor family, ELF3 has been reported to be involved in a number of different types of cancer, including colon, ovarian and ampullary cancers, but the role of ELF3 in other types of tumors is still controversial (19-21). Indeed, a number of studies have revealed multifunctional roles and different mechanisms of ELF3 in cancer $(10,11,19-22)$. It is clear that ETS factors such as ETS-1 and ETS-2 serve a critical role in thyroid cell transformation (23). However, the role and mechanisms of ELF3 in thyroid cancer have remained unknown until now. The present study investigated the biological role of ELF3 in thyroid cancer cells with a series of in vitro studies. As expected, ELF3 knockdown resulted in a strong inhibition of growth via suppression of cell proliferative and colony forming capacity. Furthermore, ELF3 knockdown reduced cell migration/invasion. Collectively, these results suggested that ELF3 possesses a strong tumorigenic function in thyroid cancer.

To better understand the mechanism of ELF3 as an oncogene in thyroid cancer, the association between ELF3 and two major cascades (MAPK/Erk and PI3K/Akt) in thyroid cancer cells was investigated. As major therapeutic targets, these two pathways serve important roles in thyroid tumorigenesis (5). On the one hand, the RAF inhibitor PLX4032 was revealed to significantly reduce the expression of ELF3 in BRAF-mutant thyroid cancer cell lines; further analysis of the TCGA dataset indicated a higher expression of ELF3 in BRAF-mutant thyroid 
A
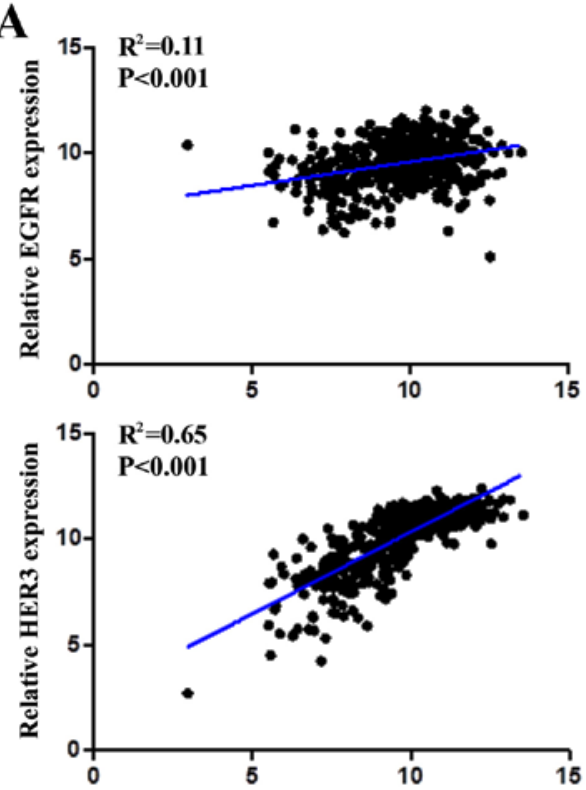
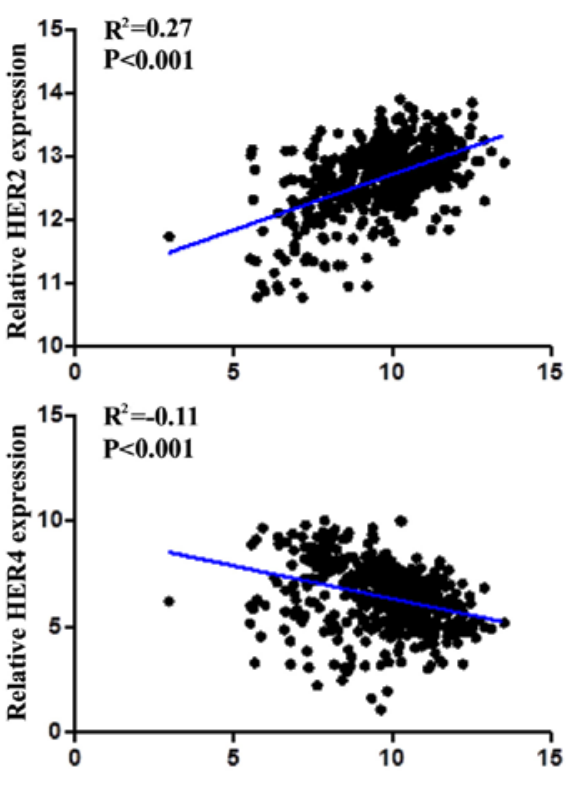

B

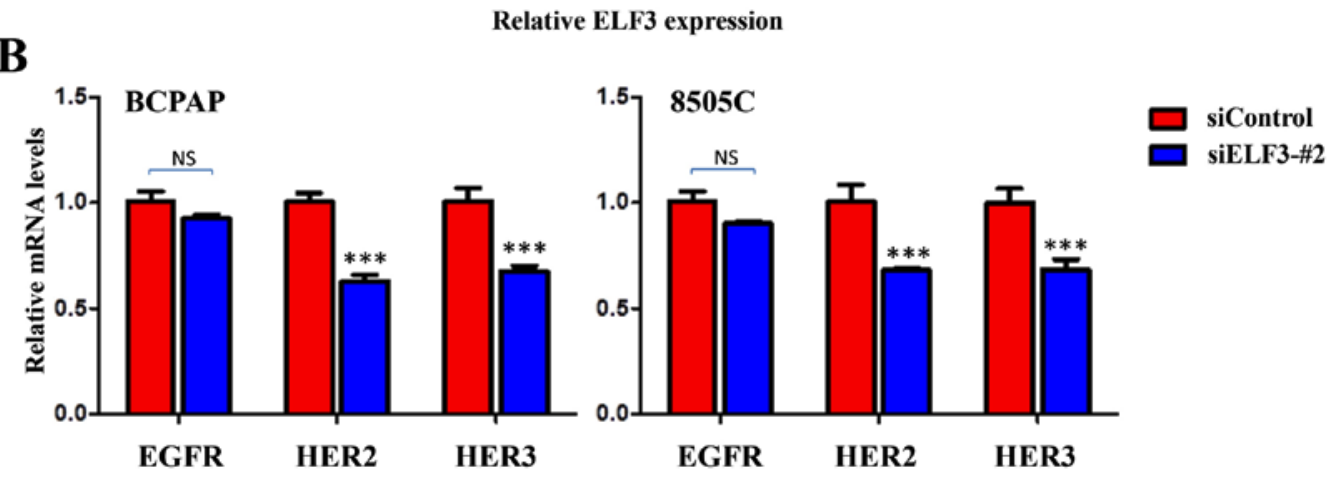

C

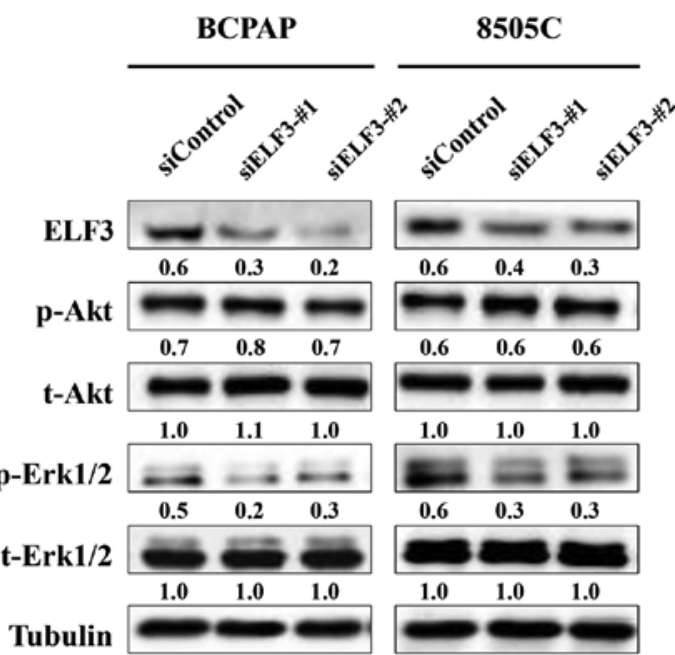

Figure 5. E26 transformation (ETS)-specific related transcription factor-3 (ELF3) transcriptionally regulates the expression of HER/ErbB family of receptors and modulates the activity of the mitogen-activated protein kinase MAPK/extracellular receptor kinase (Erk) pathway. (A) The correlation between mRNA expression of ELF3 and HER receptors (EGFR, HER2, HER3 and HER4) in primary PTC from the TCGA cohort (n=505). (B) RT-qPCR assay was performed to investigate the effect of ELF3 silencing on the expression of EGFR, HER2 and HER3. Expression levels of these genes were normalized with 18S rRNA levels. (C) Effects of ELF3 knockdown on the MAPK/Erk pathway and phosphatidylinositol-3-kinase (PI3K)/protein kinase B (Akt) pathway were assessed by western blot analysis. Tubulin was used as a loading control. Experiments were performed $>3$ times. All the data are presented as mean \pm standard deviation. ${ }^{* * * *} \mathrm{P}<0.001$. NS, not significant.

cancer. On the other hand, knockdown of ELF3 strongly reduced the phosphorylation of Erk, supporting the hypothesis that ELF3 performs its oncogenic function in thyroid cancer through modulation of the activity of the MAPK/Erk pathway and the formation of positive feedback loops with the MAPK/Erk pathway. However, the mechanism underlying the 
upregulation of ELF3 by the MAPK/Erk signaling pathway warramts further investigation.

The proximal HER family member promoter contains a conserved ETS-responsive element (GAGGAA), which is recognized by an ETS-immunoreactive factor in breast cancer cells (24). Although $>10$ different ETS transcription factors have been revealed in human cancers, only a few ETS family members including ELF3 $(25,26)$ have been reported as potential HER family member transactivators. The HER/ErbB family of receptors are transmembrane receptor tyrosine kinases that were demonstrated to be widely overexpressed in a variety of human cancers including thyroid cancer $(27,28)$. Furthermore, the constitutively activated HER/ErbB family of receptors promotes cell proliferation and inhibits apoptosis via the MAPK/Erk pathway (29). Further analysis of the TGCA database indicated positive associations of ELF3 expression with the expression of EGFR, HER2 and HER3. In addition, knockdown of ELF3 in thyroid cancer cells significantly reduced the expression of EGFR, HER2 and HER3, respectively. These data suggested that the HER/ErbB family of receptors may be a potential downstream target of ELF3. It was therefore hypothesized that ELF3 formed positive feedback loops with MAPK/Erk pathway through transcriptional regulation of HER2 and HER3 expression. Although some potential ETS binding sites were found in HER/ErbB family promoter regions with MatInspector online software, further investigations are required to investigate the specific ELF3 binding site within the HER/ErbB family promoter regions. Notably, no influence of ELF3 knockdown on the PI3K/Akt pathway was revealed; this pathway is located downstream of the HER/ErbB family and has been revealed to serve an important role in thyroid tumorigenesis $(5,18)$. This may be due to differences in the expression of different components in these signaling pathways, including Smad4, which was proven to serve a crucial role in connecting ELF3 and the PI3K/Akt pathway (30). Further studies are required to better understand the mechanism underlying the associations between ELF3 and the PI3K/Akt pathway in thyroid cancer. In conclusion, overexpression of ELF3 was demonstrated to be a potential prognostic marker in patients with thyroid cancer. ELF3 transcriptionally regulates the expression of the HER/ErbB family of receptors and forms a positive feedback loop with the MAPK pathway leading to the progression of BRAF-mutant thyroid cancer. ELF3 may function as a possible therapeutic target against thyroid cancer.

\section{Acknowledgements}

This research was supported by the Department of Endocrinology, Ankang City Central Hospital.

\section{Funding}

No funding was received.

\section{Availability of data and materials}

The datasets used during the current study are available from the corresponding author on reasonable request.

\section{Authors' contributions}

JK designed the research. $\mathrm{HC}$ and $\mathrm{XZ}$ performed the research and drafted the manuscript. HC, ZW, WC, XZ, LH, GT and $\mathrm{JK}$ analyzed the data and were also involved in writing the manuscript. All authors read and approved the manuscript and agree to be accountable for all aspects of the research in ensuring that the accuracy or integrity of any part of the work are appropriately investigated and resolved.

\section{Ethics approval and consent to participate}

Written informed consent was obtained from all of the patients before surgery. This study was approved by the Ethics Committee of Ankang Central Hospital and Zhoukou Central Hospital.

\section{Patient consent for publication}

Not applicable.

\section{Competing interests}

The authors declare that they have no competing interests.

\section{References}

1. Chen AY, Jemal A and Ward EM: Increasing incidence of differentiated thyroid cancer in the United States, 1988-2005. Cancer 115: 3801-3807, 2009.

2. Siegel RL, Miller KD and Jemal A: Cancer statistics, 2015. CA Cancer J Clin 65: 5-29, 2015.

3. Hay ID, Thompson GB, Grant CS, Bergstralh EJ, Dvorak CE, Gorman CA, Maurer MS, McIver B, Mullan BP, Oberg AL, et al: Papillary thyroid carcinoma managed at the Mayo Clinic during six decades (1940-1999): Temporal trends in initial therapy and long-term outcome in 2444 consecutively treated patients. World J Surg 26: 879-885, 2002

4. Burns WR and Zeiger MA: Differentiated thyroid cancer. Semin Oncol 37: 557-566, 2010.

5. Xing M: Molecular pathogenesis and mechanisms of thyroid cancer. Nat Rev Cancer 13: 184-199, 2013.

6. Nikiforov YE and Nikiforova MN: Molecular genetics and diagnosis of thyroid cancer. Nat Rev Endocrinol 7: 569-580, 2011

7. Cancer Genome Atlas Research Network: Integrated genomic characterization of papillary thyroid carcinoma. Cell 159: 676-690, 2014.

8. Oikawa T and Yamada T: Molecular biology of the Ets family of transcription factors. Gene 303: 11-34, 2003.

9. Oliver JR, Kushwah R and Hu J: Multiple roles of the epithelium-specific ETS transcription factor, ESE-1, in development and disease. Lab Invest 92: 320-330, 2012.

10. Longoni N, Sarti M, Albino D, Civenni G, Malek A, Ortelli E, Pinton S, Mello-Grand M, Ostano P, D'Ambrosio G, et al: ETS transcription factor ESE1/ELF3 orchestrates a positive feedback loop that constitutively activates NF- $\kappa \mathrm{B}$ and drives prostate cancer progression. Cancer Res 73: 4533-4547, 2013.

11. Gajulapalli VN,Samanthapudi VS,Pulaganti M,Khumukcham SS, Malisetty VL, Guruprasad L, Chitta SK and Manavathi B: A transcriptional repressive role for epithelial-specific ETS factor ELF3 on oestrogen receptor alpha in breast cancer cells. Biochem J 473: 1047-1061, 2016.

12. Livak KJ and Schmittgen TD: Analysis of relative gene expression data using real-time quantitative PCR and the $2^{-\Delta \Delta C_{\mathrm{T}}}$ method. Methods 25: 402-408, 2001.

13. Cancer Genome Atlas Research Network; Weinstein JN, Collisson EA, Mills GB, Shaw KR, Ozenberger BA, Ellrott K, Shmulevich I, Sander C and Stuart JM: The cancer genome atlas pan-cancer analysis project. Nat Genet 45: 1113-1120, 2013.

14. Jemal A, Bray F, Center MM, Ferlay J, Ward E and Forman D: Global cancer statistics. CA Cancer J Clin 61: 69-90, 2011. 
15. Haugen DR, Akslen LA, Varhaug JE and Lillehaug JR: Expression of c-erbB-2 protein in papillary thyroid carcinomas. Br J Cancer 65: 832-837, 1992.

16. Haugen DR, Akslen LA, Varhaug JE and Lillehaug JR: Expression of c-erbB-3 and c-erbB-4 proteins in papillary thyroid carcinomas. Cancer Res 56: 1184-1188, 1996.

17. Mitsiades CS, Kotoula V, Poulaki V, Sozopoulos E, Negri J, Charalambous E, Fanourakis G, Voutsinas G, Tseleni-Balafouta S and Mitsiades N: Epidermal growth factor receptor as a therapeutic target in human thyroid carcinoma: Mutational and functional analysis. J Clin Endocrinol Metab 91: 3662-3666, 2006.

18. Lemmon MA and Schlessinger J: Cell signaling by receptor tyrosine kinases. Cell 141: 1117-1134, 2010.

19. Wang JL, Chen ZF, Chen HM, Wang MY, Kong X, Wang YC, Sun TT, Hong J, Zou W, Xu J, et al: Elf3 drives $\beta$-catenin transactivation and associates with poor prognosis in colorectal cancer. Cell Death Dis 5: e1263, 2014.

20. Yeung TL, Leung CS, Wong KK, Gutierrez-Hartmann A, Kwong J, Gershenson DM and Mok SC: ELF3 is a negative regulator of epithelial-mesenchymal transition in ovarian cancer cells. Oncotarget 8: 16951-16963, 2017.

21. Gingras MC, Covington KR, Chang DK, Donehower LA, Gill AJ, Ittmann MM, Creighton CJ, Johns AL, Shinbrot E, Dewal N, et al: Ampullary cancers harbor ELF3 tumor suppressor gene mutations and exhibit frequent WNT dysregulation. Cell Rep 14: 907-919, 2016.

22. Yachida S, Wood LD, Suzuki M, Takai E, Totoki Y, Kato M, Luchini C, Arai Y, Nakamura H, Hama N, et al: Genomic sequencing identifies $E L F 3$ as a driver of ampullary carcinoma. Cancer Cell 29: 229-240, 2016.
23. de Nigris $F$, Mega T, Berger $N$, Barone MV, Santoro $M$, Viglietto G, Verde P and Fusco A: Induction of ETS-1 and ETS-2 transcription factors is required for thyroid cell transformation. Cancer Res 61: 2267-2275, 2001.

24. Scott GK, Daniel JC, Xiong X, Maki RA, Kabat D and Benz CC: Binding of an ETS-related protein within the DNase I hypersensitive site of the HER2/neu promoter in human breast cancer cells. J Biol Chem 269: 19848-19858, 1994.

25. Chang CH, Scott GK, Kuo WL, Xiong X, Suzdaltseva Y, Park JW, Sayre P, Erny K, Collins C, Gray JW, et al: ESX: A structurally unique Ets overexpressed early during human breast tumorigenesis. Oncogene 14: 1617-1622, 1997.

26. Eckel KL, Tentler JJ, Cappetta GJ, Diamond SE and Gutierrez-Hartmann A: The epithelial-specific ETS transcription factor ESX/ESE-1/Elf-3 modulates breast cancer-associated gene expression. DNA Cell Biol 22: 79-94, 2003.

27. Fisher KE, Jani JC, Fisher SB, Foulks C, Hill CE, Weber CJ, Cohen $\mathrm{C}$ and Sharma J: Epidermal growth factor receptor overexpression is a marker for adverse pathologic features in papillary thyroid carcinoma. J Surg Res 185: 217-224, 2013.

28. Landriscina M, Pannone G, Piscazzi A, Toti P, Fabiano A, Tortorella S, Occhini R, Ambrosi A, Bufo P and Cignarelli M: Epidermal growth factor receptor 1 expression is upregulated in undifferentiated thyroid carcinomas in humans. Thyroid 21: 1227-1234, 2011.

29. Kolch W and Pitt A: Functional proteomics to dissect tyrosine kinase signalling pathways in cancer. Nat Rev Cancer 10: 618-629, 2010.

30. Liu J, Cho SN, Akkanti B, Jin N, Mao J, Long W, Chen T, Zhang Y, Tang X, Wistub II, et al: ErbB2 pathway activation upon Smad4 loss promotes lung tumor growth and metastasis. Cell Rep: Mar 3, 2015 (Epub ahead of print). 